\title{
Complication rates as a trauma care performance indicator: a systematic review
}

Lynne Moore ${ }^{1,2^{*}}$, Henry Thomas Stelfox ${ }^{2}$ and Alexis F Turgeon 3,4

\begin{abstract}
Introduction: Information on complication rates is essential to trauma quality improvement efforts. However, it is unclear which complications are the most clinically relevant. The objective of this study was to evaluate whether there is consensus on the complications that should be used to evaluate the performance of acute care trauma hospitals.

Methods: We searched the Medline, EMBASE, Cochrane Central, CINAHL, BIOSIS, TRIP and ProQuest databases and included studies using at least one nonfatal outcome to evaluate the performance of acute care trauma hospitals. Data were extracted in duplicate using a piloted electronic data abstraction form. Consensus was considered to be reached if a specific complication was used in $\geq 70 \%$ of studies (strong recommendation) or in $\geq 50 \%$ of studies (weak recommendation).

Results: Of 14,521 citations identified, 22 were eligible for inclusion. We observed important heterogeneity in the complications used to evaluate trauma care. Seventy-nine specific complications were identified but none were used in $\geq 70 \%$ of studies and only three (pulmonary embolism, deep vein thrombosis, and pneumonia) were used in $\geq 50 \%$ of studies. Only one study provided evidence for the clinical relevance of complications used and only five studies (23\%) were considered of high methodological quality.

Conclusion: Based on the results of this review, we can make a weak recommendation on three complications that should be used to evaluate acute care trauma hospitals; pulmonary embolism, deep vein thrombosis, and pneumonia. However, considering the observed disparity in definitions, the lack of clinical justification for the complications used, and the low methodological quality of studies, further research is needed to develop a valid and reliable performance indicator based on complications that can be used to improve the quality and efficiency of trauma care.
\end{abstract}

\section{Introduction}

Complications following admission for traumatic injury are common and have been shown to increase hospital mortality, length of stay, and costs [1-5]. These complications have been associated with a negative impact on long-term functional capacity and quality of life [6]. Many complications are potentially avoidable and quality improvement strategies aimed at reducing them have been shown to have a positive impact on patient outcome and resource utilization $[7,8]$.

Complications have been identified as a priority for the development of trauma performance indicators

\footnotetext{
* Correspondence: lynne.moore.cha@ssss.gouv.qc.ca

'Department of Social and Preventive Medicine, Université Laval, 2325 rue

de I'Université, Québec City, Québec, Canada GIV OA6

Full list of author information is available at the end of the article
}

$[9,10]$. Furthermore, US Trauma Quality Improvement Program members have made efforts to track information on complications following trauma using the National Trauma Data Bank according to the National Surgical Quality Improvement program [11,12]. However, despite the widespread availability of routinely collected data on hospital complications, a performance indicator based on complications has yet to be validated specifically in the context of acute trauma care $[9,10]$. Considering the potential positive and negative consequences of healthcare performance evaluation [13], such a performance indicator should be based on clinically relevant complications identified using standardized definitions [14] and robust methodology including adequate risk adjustment [15].

\section{Biomed Central}


A recent systematic review synthesized studies evaluating performance indicators in trauma care [9]. However, information specific to the assessment of complications in the context of acute trauma care is limited. The objective of this study was to evaluate whether there is consensus on the complications that should be used to evaluate the performance of acute care trauma hospitals.

\section{Materials and methods}

We designed a systematic review of cohort studies evaluating the performance of acute care hospitals for the treatment of general trauma populations using information on complications. This systematic review was conducted following recommendations from the Cochrane handbook for systematic reviews [16] and in compliance with the Preferred Reporting Items for Systematic Reviews and Meta-Analyses (PRISMA) statement [17]. The study was approved by our institutional research ethics committee. The present systematic review complements a review published by one of the authors (HTS) in 2011. The latter review was designed to document evidence of the reliability and validity of trauma quality indicators at large, whereas the present study is designed to evaluate whether there is consensus on the complications used to evaluate trauma care quality.

\section{Search strategy}

We searched the Medline, EMBASE, Cochrane Library, CINAHL, BIOSIS, TRIP and ProQuest databases for peer-reviewed articles and postgraduate academic publications. The websites of the following trauma associations were also searched: American College of Surgeons, American Association for the Surgery of Trauma, Eastern Association for the Surgery of Trauma, American Trauma Society, British Trauma Society, Trauma Association of Canada, Australasian Trauma Society, Western Trauma Association, Trauma.org, Society of Trauma Nurses, and International Trauma Anaesthesia and Critical Care Society. Our initial systematic search strategy was based on nonfatal outcomes at large. We then selected studies based on complications for this systematic review. The search strategy was designed for Medline and EMBASE using keywords and MeSH (Medline) or Emtree (EMBASE) for three groups of terms: trauma, performance, and nonfatal outcomes (see Additional file 1). Keywords were elaborated by a group of experts with methodological and clinical expertise who are co-investigators and collaborators on the project. The systematic search was limited to human studies from high-income countries conducted from the earliest available date of the search engine to 1 July 2011. The bibliographies for all identified studies and reviews were searched manually to identify additional relevant publications. No publication type, year, or language restriction was used.

\section{Study selection}

Both prospective and retrospective cohort studies evaluating the performance of acute care hospitals for the treatment of global trauma populations using at least one medical complication were considered eligible. A complication was defined as an additional problem that arises following a procedure, treatment, or illness and is secondary to it [18].

Duplicates were identified and sorted by two independent reviewers with methodological and content expertise (LM and HTS) using EndNote software version X4 (2010; Thomson Reuters, New York, NY, USA). These two reviewers independently evaluated citations identified for eligibility by screening titles, abstracts, and full publications. Disagreement on study eligibility was resolved by consensus, and a third reviewer (AFT) was involved when required. Inter-rater agreement was evaluated with kappa statistics on study eligibility. Articles written in a language other than English were translated.

\section{Data abstraction}

The same two reviewers independently extracted data using a standardized data abstraction form, which was piloted on a sample of five representative studies. The data abstraction form was designed to capture information on the study setting and design, complications, and methodological quality. The latter was evaluated using elements selected from the following sources: a tool proposed to evaluate the quality of complication-reporting in the surgical literature [14], the Strengthening the Reporting of Observational Studies in Epidemiology statement [19], the Cochrane risk of bias tool [20], and the Downs and Black tool [21]. The following 10 methodological quality criteria were thus selected by the project steering committee based on the consideration that they are important for the validity and reliability of performance evaluations: definitions of complications provided; justification for the choice of specific complications provided; duration of follow-up indicated; number and percentage of specific complications indicated; severity of complications considered (for example, major versus minor complications); risk adjustment used; data quality assurance efforts reported; adequate treatment of missing data; estimates of variation given; and adequate sample size.

Adequate treatment of missing data implied that the absence/presence and proportion of missing data were reported, and if $>10 \%$ of subjects had missing data then imputation techniques that take account of the uncertainty of missing data values (for example, multiple imputation/maximum expectation) or sensitivity analysis were used [22]. Sample size was considered adequate if at least 100 patients per hospital were available for analysis; if not, analysis strategies designed for low-volume centers (for example, shrinkage techniques) were used [15]. 
Disagreement on abstracted data between reviewers was resolved by consensus or, if necessary, consultation with a third reviewer (AFT).

\section{Analysis}

A classification system for complications following traumatic injury proposed elsewhere [23] was used to categorize specific complications identified in this review. Complications were thus grouped into the following 10 categories: pulmonary, cardiovascular, gastrointestinal, hepatic/biliary, hematologic, infection, genitourinary/renal, musculoskeletal/integumentary, neurologic, vascular, psychiatric, and other. According to Cochrane GRADE criteria for clinical recommendations [24], consensus was considered to be reached for a particular complication if at least $70 \%$ (strong recommendation) or $50 \%$ (weak recommendation) of studies used that complication to evaluate trauma care. Sensitivity analyses were performed to see whether greater consensus was achieved in more recent studies (later than 2005). Studies were considered to be of high methodology quality if at least seven out of 10 quality criteria were respected [25].

\section{Results}

\section{Search results}

We identified 14,521 citations, of which 112 were selected for full text review after screening of titles and abstracts (Table 1). A total of 22 studies met the inclusion criteria [26-47]. We had excellent inter-rater agreement on the selection of eligible studies with a kappa statistic of 0.89 (95\% confidence interval $=0.79$ to 0.99 ).

\section{Study characteristics}

Of 22 selected studies, 20 (91\%) were conducted in the USA [26,27,29-31,33-47], one in Europe [28], and one in Australia [32]. The studies spanned data collected from 1990 [26] through 2007 [35,36,38]. The majority of studies were based on a single trauma center $(n=15)$ [26,30-34,37-39,41,43-47], six were based on national (USA) trauma data $[27,29,35,36,40,42]$, and one was based on the comparison of two trauma centers [28]. All but two studies $[27,47]$ were retrospective cohort studies. The mean Injury Severity Score varied between 8 and 29 $[26,28,30,31,33,34,37,38,40,41,45,46]$. The proportion of blunt trauma varied between 52\% [37] and 97\% [43]. Six studies were restricted to adult trauma patients $[27,29,32,36,37,40]$, while none of the other studies mentioned inclusion or exclusion criteria based on age.

\section{Complications}

Four studies (18\%) did not provide any information on the specific complications used to evaluate performance $[31,32,38,44]$. Among the other studies, we observed important heterogeneity in the complications used
(Table 2). A total of 79 different complications were identified. None of the complications were used in more than $70 \%$ of studies (consistent with a strong recommendation) but the following complications were used in at least $50 \%$ of studies (weak recommendation): pulmonary embolism $(n=12)$, deep vein thrombosis $(n=$ $12)$, and pneumonia $(n=11)$. Other commonly evaluated complications were sepsis $(n=8)$, urinary tract infection $(n=8)$, renal failure $(n=7)$, myocardial infarction $(n=7)$, wound infection $(n=7)$, decubitus ulcer $(n=6)$, respiratory failure $(n=6)$, and acute respiratory distress syndrome $(n=6)$.

\section{Sensitivity analyses}

Among 11 studies published later than 2005 [27-29,33,35-37,40-42,46], we observed similar results; pulmonary embolism, deep vein thrombosis, and pneumonia were used in more than $50 \%$ of studies.

\section{Methodological quality}

Only five studies (23\%) were considered of high methodological quality with at least seven out of 10 quality criteria respected (Table 3). Eight studies gave no definition of the complications used to evaluate performance (Table 3) $[31,32,34,37,38,41,44,46]$. Six studies gave medical $(n=3)$ $[27,35,47]$ or diagnostic code $(n=4)[30,33,40,42]$ definitions of specific complications, whereas seven studies provided a reference for the definition of selected complications $[26,28,29,36,39,43,45]$. Thirteen studies (59\%) gave no justification for their choice of complications $[27,30-35,37,38,41,44,46,47]$. Only one study justified the clinical relevance of included complications [36]; the authors selected eight complications reported to have the highest attributable mortality in a trauma population [1]. Three studies used certain complications recommended for quality improvement activities by the US Agency for Health Care Research and Quality [29,40,42]. Two studies used certain complications identified by the American College of Surgeons Committee on Trauma [28,45]. Two studies used complications observed in an independent trauma population [26,39], and one study [43] was based on complications used to evaluate quality in a general admission population [15].

Among the 22 studies included, six (15\%) indicated the duration of follow-up $[26,35,36,40,42,44]$, which was always until hospital discharge. The number and proportion of each specific complication were given in 11 studies [27,36-38,40-42,44-47]. The severity of complications (that is, major versus minor) was specified in three studies [34-36]. Nearly all studies had adequate sample size to evaluate performance, and $50 \%$ presented estimates of variation (that is, standard errors or confidence intervals). However, less than one-half of the studies used risk adjustment, one study reported using data 
Table 1 Description of included studies

\begin{tabular}{|c|c|c|c|c|c|c|c|c|c|}
\hline Author, year (reference) & Setting & Study design & Period & Patients $(n)$ & Centers $(n)$ & Age (years) & Mechanism (\% blunt) & Gender (\% male) & ISS \\
\hline Ang and colleagues, 2009 [27] & NSCOT, USA & Prospective & 2001 to 2002 & 5,043 & 69 & $29 \% \geq 65$ & 88 & 62 & $74 \% \geq 16$ \\
\hline Calderale and colleagues, 2008 [28] & Level I TC, Italy/Romania & Retrospective & 2002 & 182 & 2 & Mean $=43$ & $74 \% \mathrm{MVC}$ & 80 & Mean $=29$ \\
\hline Chang and colleagues, 2008 [29] & NIS, USA & Retrospective & 2000 to 2004 & $1,350,229$ & NA & Mean $=60$ & NR & 48 & Median $=8$ \\
\hline Claridge and colleagues, 2001 [30] & Level I TC, USA & Retrospective & 1994 to 1999 & 917 & 1 & Mean $=41$ & 88 & 67 & Mean $=14$ \\
\hline Cohen and colleagues, 1999 [31] & Level I TC, USA & Retrospective & 1995 to 1997 & 1,025 & 1 & Mean $=43$ & 75 & 66 & Mean $=9$ \\
\hline Curtis and colleagues, 2002 [32] & TC, Australia & Retrospective & 2000 to 2001 & 475 & 1 & NR & NR & NR & NR \\
\hline Davis and colleagues, 2008 [33] & Level I TC, USA & Retrospective & 2005 to 2006 & 1,058 & 1 & NR & 85 & NR & Mean $=13$ \\
\hline DiRusso and colleagues, 2001 [34] & Level I TC, USA & Retrospective & 1994 and 1998 & 2,774 & 1 & Mean $=36$ & 94 & 57 & Mean $=11$ \\
\hline Glance and colleagues, 2011 [35] & NTDB, USA & Retrospective & 2007 & 54,713 & 42 & Median $=37$ & 91 & 67 & NR \\
\hline Haas and colleagues, 2011 [36] & NTDB, USA & Retrospective & 2007 & 76,048 & 115 & Mean $=45$ & 90 & 69 & $49 \%>15$ \\
\hline Haut and colleagues, 2007 [37] & Level I TC, USA & Retrospective & 1995 to 2005 & 7,559 & 1 & Mean $=33$ & 52 & 80 & Mean $=10$ \\
\hline Highstead and colleagues, 2009 [38] & Level I TC, USA & Retrospective & 1998 to 2007 & 7,593 & 1 & Mean $=36$ & 78 & 76 & Mean $=8$ \\
\hline Hinsdale and colleagues, 1998 [39] & TC, USA & Retrospective & 1993 to 1996 & 6,992 & 1 & NR & NR & NA & NR \\
\hline Hoyt and colleagues, 2003 [26] & Level I TC, USA & Retrospective & 1990 to 2001 & 13,382 & 1 & NR & 71 & 78 & Mean $=13$ \\
\hline Huseynova and colleagues, 2009 [40] & NTDB, USA & Retrospective & 2006 & 22,421 & 30 & Mean $=45$ & 90 & 68 & Mean $=17$ \\
\hline Jacobs and colleagues, 2009 [41] & Level I TC, USA & Retrospective & 2006 & 1,959 & 1 & Mean $=37$ & 82 & 72 & Mean $=15$ \\
\hline Pierce and colleagues, 2008 [42] & NTDB, USA & Retrospective & 2001 to 2005 & 578,252 & 147 & NR & NR & NA & NR \\
\hline Piontek and colleagues, 2003 [43] & Level I TC, USA & Retrospective & 1993 to 2001 & 7,811 & 1 & NR & 97 & NR & NR \\
\hline Podnos and colleagues, 1998 [44] & Level I TC, USA & Retrospective & 1996 & 1,427 & 1 & NR & NR & 73 & NR \\
\hline Roettger and colleagues, 2005 [45] & Level I TC, USA & Retrospective & 2001 to 2004 & 1,391 & 1 & Mean $=37$ & 87 & 66 & Mean $=11$ \\
\hline Rotondo and colleagues, 2009 [46] & Level I TC, USA & Retrospective & 1994 to 2005 & 18,644 & 1 & Mean $=41$ & 88 & 63 & Mean $=11$ \\
\hline Schuerer and colleagues, 2005 [47] & Level I TC, USA & Prospective & 2002 to 2003 & 2,531 & 1 & Mean $=39$ & NR & NR & Median $=9$ \\
\hline
\end{tabular}

ISS, Injury Severity Score; MVC, motor vehicle collision; NA, not applicable; NIS, National Inpatient Survey; NR, not reported; NSCOT, National Study on Costs and Outcomes of Trauma; NTDB, National Trauma Data Bank; TC, trauma center; 
Table 2 Identification of acute care medical complications

\begin{tabular}{|c|c|c|c|c|c|c|c|c|c|c|c|c|c|c|c|c|c|c|}
\hline & \multicolumn{18}{|c|}{ Reference } \\
\hline & {$[27]$} & {$[28]$} & {$[29]$} & {$[30]$} & {$[33]$} & {$[34]$} & {$[35]$} & {$[36]$} & {$[37]$} & {$[39]$} & {$[26]$} & {$[40]$} & {$[41]$} & {$[42]$} & {$[43]$} & {$[45]$} & {$[46]$} & {$[47]$} \\
\hline \multicolumn{19}{|l|}{ Pulmonary } \\
\hline Pulmonary embolism ${ }^{\mathrm{a}}$ & $x$ & $x$ & $x$ & & & $x$ & $x$ & & $x$ & $x$ & $x$ & $x$ & & & & $x$ & $x$ & $x$ \\
\hline Pneumonia $^{a}$ & $x$ & & & $x$ & $x$ & $x$ & $x$ & $x$ & & $x$ & $x$ & & $x$ & & $x$ & $x$ & & \\
\hline ARDS & & & & & & & $x$ & $x$ & & $x$ & $x$ & & $x$ & & & $x$ & & \\
\hline Respiratory failure & & & & & & $x$ & & & & $x$ & $x$ & & $x$ & & & $x$ & $x$ & \\
\hline Hemothorax/pneumothorax & & & $x$ & & & & & & & $x$ & $x$ & & & & & $x$ & & \\
\hline Aspiration/pneumonia & & & & & & & & & & $x$ & $x$ & & & & & $x$ & & \\
\hline Empyema & & & & & & & & & & $x$ & $x$ & & & & & $x$ & & \\
\hline Pleural effusion & & & & & & & & & & $x$ & $x$ & & $x$ & & & & & \\
\hline Abscess & & & & & & & & & & $x$ & $x$ & & & & & & & \\
\hline Atelectasis & & & & & & & & & & $x$ & $x$ & & & & & & & \\
\hline Fat embolus & & & & & & & & & & $x$ & $x$ & & & & & & & \\
\hline Pulmonary edema & & & & & & & & & & $x$ & $x$ & & & & & & & \\
\hline Respiratory failure/distress & & & & & & & & & & $x$ & $x$ & & & & & & & \\
\hline \multicolumn{19}{|l|}{ Infections } \\
\hline Sepsis & $x$ & & & & $x$ & $x$ & $x$ & $x$ & & $x$ & $x$ & & $x$ & & & & & \\
\hline Wound & $x$ & & & $x$ & & & & & & $x$ & $x$ & & $x$ & & $x$ & $x$ & & \\
\hline Sepsis-like syndrome & & & & & & & & & & $x$ & $x$ & & $x$ & & & $x$ & & \\
\hline Catheter-related (line infection) & & & & $x$ & & & & & & $x$ & $x$ & & & & & & & \\
\hline Disseminated fungal & & & & & & & & & & $x$ & $x$ & & & & & $x$ & & \\
\hline Intra abdominal & & & & & & & & & & $x$ & $x$ & & & & & $x$ & & \\
\hline Sinusitis & & & & & & & & & & $x$ & $x$ & & & & & $x$ & & \\
\hline Cellulitis/traumatic & & & & & & & & & & $x$ & $x$ & & & & & & & \\
\hline Necrotizing fascitis & & & & & & & & & & $x$ & $x$ & & & & & & & \\
\hline Yeast & & & & & & & & & & $x$ & $x$ & & & & & & & \\
\hline Bloodstream & & & & $x$ & & & & & & & & & & & & & & \\
\hline Postoperative & & & & & & & & & & & & & & & $x$ & & & \\
\hline \multicolumn{19}{|l|}{ Vascular } \\
\hline Deep vein thrombosis ${ }^{a}$ & $x$ & $x$ & $x$ & & & & & & $x$ & $x$ & $x$ & $x$ & & $x$ & $x$ & $x$ & $x$ & $x$ \\
\hline Anastomosis hemorrhage & & & & & & & & & & $x$ & $x$ & & & & & & & \\
\hline Embolus (nonpulmonary) & & & & & & & & & & $x$ & $x$ & & & & & & & \\
\hline Gangrene & & & & & & & & & & $x$ & $x$ & & & & & & & \\
\hline Graft infection & & & & & & & & & & $x$ & $x$ & & & & & & & \\
\hline Thrombosis & & & & & & & & & & $x$ & $x$ & & & & & & & \\
\hline \multicolumn{19}{|l|}{ Genitourinary/renal } \\
\hline Urinary tract infection & $x$ & & & $x$ & $x$ & $x$ & & & & $x$ & $x$ & & $x$ & & & $x$ & & \\
\hline Renal failure & & & & & & $x$ & $x$ & $x$ & & $x$ & $x$ & & $x$ & & & $x$ & & \\
\hline Urethral injury & & & & & & & & & & $x$ & $x$ & & & & & & & \\
\hline \multicolumn{19}{|l|}{ Cardiovascular } \\
\hline Myocardial infarction & & & & & & & $x$ & $x$ & & $x$ & $x$ & & & & $x$ & $x$ & $x$ & \\
\hline Arrhythmia & $x$ & & & & & & & & & $x$ & $x$ & & $x$ & & & $x$ & & \\
\hline Cardiac arrest & $x$ & & & & & & & $x$ & & $x$ & $x$ & & & & & $x$ & & \\
\hline Shock & & & & & & & & & & $x$ & $x$ & & & & $x$ & $x$ & & \\
\hline Congestive heart failure & & & & & & & & & & $x$ & $x$ & & & & & $x$ & & \\
\hline Cardiogenic shock & & & & & & & & & & $x$ & $x$ & & & & & & & \\
\hline Pericardial effusion or tamponade & & & & & & & & & & $x$ & $x$ & & & & & & & \\
\hline Pericarditis & & & & & & & & & & $x$ & $x$ & & & & & & & \\
\hline \multicolumn{19}{|l|}{ Neurologic } \\
\hline Stroke & & & & & & & $x$ & $x$ & & $x$ & $x$ & & & & & & $x$ & \\
\hline Progression of original neurologic insult & & & & & & & & & & $x$ & $x$ & & & & & $x$ & & \\
\hline
\end{tabular}


Table 2 Identification of acute care medical complications (Continued)

\begin{tabular}{|c|c|c|c|c|c|c|c|}
\hline Alcohol withdrawal & & & & $x$ & $x$ & & \\
\hline Anoxic encephalopathy & & & & $x$ & $x$ & & \\
\hline Diabetes insipidus & & & & $x$ & $x$ & & \\
\hline Meningitis & & & & $x$ & $x$ & & \\
\hline Neuropraxia (iatrogenic) & & & & $x$ & $x$ & & \\
\hline Nonoperative SDH/EDH & & & & $x$ & $x$ & & \\
\hline Seizure in hosp & & & & $x$ & $x$ & & \\
\hline SIADH & & & & $x$ & $x$ & & \\
\hline Ventriculitis & & & & $x$ & $x$ & & \\
\hline \multicolumn{8}{|l|}{ Gastrointestinal } \\
\hline Evisceration/dehiscence & & & & $x$ & $x$ & & $x$ \\
\hline Gastro-intestinal fistula & & & & $x$ & $x$ & & $x$ \\
\hline Peritonitis & & $x$ & & $x$ & $x$ & & \\
\hline Small bowel obstruction & & & & $x$ & $x$ & & $x$ \\
\hline Abdominal compartment syndrome & & & $x$ & & & & $x$ \\
\hline Anastomic leak & & & & $x$ & $x$ & & \\
\hline Bowel injury (iatrogenic) & & & & $x$ & $x$ & & \\
\hline Hemorrhage & & & & $x$ & $x$ & & \\
\hline Ileus & & & & $x$ & $x$ & & \\
\hline Ulcer-duodenal/gastric & & & & $x$ & $x$ & & \\
\hline Gastrointestinal bleeding/stress ulceration & & & & & & & x \\
\hline \multicolumn{8}{|l|}{ Enterotomy } \\
\hline \multicolumn{8}{|l|}{ Hematologic } \\
\hline Coagulopathy & $x$ & & $x$ & $x$ & $x$ & & X \\
\hline Transfusion complication & & $x$ & & $x$ & $x$ & & \\
\hline \multicolumn{8}{|l|}{ Musculoskeletal/integumentary } \\
\hline Decubitus ulcer (skin breakdown) & $x$ & $x$ & & $x$ & $x$ & $x$ & x \\
\hline Extremity compartment syndrome & & & & $x$ & $x$ & & X \\
\hline Nonunion & & & & $x$ & $x$ & & \\
\hline Osteomyelitis & & & & $x$ & $x$ & & \\
\hline \multicolumn{8}{|l|}{ Hepatic/biliary } \\
\hline Hepatic (liver) failure & & & & $x$ & $x$ & & $x$ \\
\hline Pancreatitis & & & & $x$ & $x$ & & X \\
\hline Acalculous cholecystitis & & & & $x$ & $x$ & & \\
\hline Hepatitis & & & & $x$ & $x$ & & \\
\hline Pancreatic fistula & & & & $x$ & $x$ & & \\
\hline Splenic injury & & & & $x$ & $x$ & & \\
\hline \multicolumn{8}{|l|}{ Psychiatric } \\
\hline Psychiatric & & & & $x$ & $x$ & & \\
\hline \multicolumn{8}{|l|}{ Other } \\
\hline Hypothermia & & & & & & & $x$ \\
\hline
\end{tabular}

ARDS, acute respiratory distress syndrome; $\mathrm{EDH}$, epidural hematoma; SDH, subdural hematoma; SIADH, syndrome of inappropriate diuretic hormone secretion. ${ }^{a}$ Complications used in at least $50 \%$ of studies (consistent with weak clinical recommendations)

quality assurance [27], and four studies adequately addressed the problem of missing data $[27,29,35,36]$.

\section{Discussion}

In this systematic review, we identified 79 specific complications that have been used to evaluate acute care trauma hospitals. None of these complications were consistent with a strong clinical recommendation but pulmonary embolism, deep vein thrombosis, and pneumonia were used in over $50 \%$ of studies and were therefore consistent with a weak clinical recommendation. Studies included in our review rarely justified the choice of complications included, and they generally had low methodological quality.

Reducing hospital complications is one of the keys to reducing morbidity, mortality, and resource utilization 
Table 3 Methodological quality of included studies

\begin{tabular}{|c|c|c|c|c|c|c|c|c|c|c|}
\hline Study & $\begin{array}{l}\text { Definitions of } \\
\text { complications }\end{array}$ & $\begin{array}{l}\text { Justification for } \\
\text { choice of } \\
\text { complications }\end{array}$ & $\begin{array}{l}\text { Duration } \\
\text { of follow- } \\
\text { up }\end{array}$ & $\begin{array}{l}\text { Number and percentage } \\
\text { of specific complications }\end{array}$ & $\begin{array}{l}\text { Complication } \\
\text { severity } \\
\text { considered }\end{array}$ & $\begin{array}{l}\text { Risk } \\
\text { adjustment }\end{array}$ & $\begin{array}{l}\text { Data quality } \\
\text { assurance } \\
\text { efforts }\end{array}$ & $\begin{array}{l}\text { Adequate } \\
\text { treatment } \\
\text { missing data }\end{array}$ & $\begin{array}{l}\text { Estimates } \\
\text { of } \\
\text { variation }\end{array}$ & $\begin{array}{l}\text { Adequate } \\
\text { sample } \\
\text { size }\end{array}$ \\
\hline $\begin{array}{l}\text { Ang and } \\
\text { colleagues }[27]^{a}\end{array}$ & $x$ & & & $x$ & & $x$ & $x$ & $x$ & $x$ & $x$ \\
\hline $\begin{array}{l}\text { Calderale and } \\
\text { colleagues [28] }\end{array}$ & $x$ & $x$ & & & & & & & & $x$ \\
\hline $\begin{array}{l}\text { Chang and } \\
\text { colleagues [29] }\end{array}$ & $x$ & $x$ & & & & $x$ & & $x$ & $x$ & $x$ \\
\hline $\begin{array}{l}\text { Claridge and } \\
\text { colleagues [30] }\end{array}$ & $x$ & & & & & & & & & $x$ \\
\hline $\begin{array}{l}\text { Cohen and } \\
\text { colleagues [31] }\end{array}$ & & & & & & & & & & $x$ \\
\hline $\begin{array}{l}\text { Curtis and } \\
\text { colleagues [32] }\end{array}$ & & & & & & & & & & $x$ \\
\hline $\begin{array}{l}\text { Davis and } \\
\text { colleagues [33] }\end{array}$ & $x$ & & & & & & & & $x$ & $x$ \\
\hline $\begin{array}{l}\text { DiRusso and } \\
\text { colleagues [34] }\end{array}$ & & & & & $x$ & & & & & $x$ \\
\hline $\begin{array}{l}\text { Glance and } \\
\text { colleagues [35] }\end{array}$ & $x$ & & $x$ & & $x$ & $x$ & & X & $x$ & $x$ \\
\hline $\begin{array}{l}\text { Haas and } \\
\text { colleagues [36] }\end{array}$ & $x$ & $x$ & $x$ & $x$ & $x$ & $x$ & & $x$ & & $x$ \\
\hline $\begin{array}{l}\text { Haut and } \\
\text { colleagues [37] }\end{array}$ & & X & & $x$ & & & & & & \\
\hline $\begin{array}{l}\text { Highstead and } \\
\text { colleagues [38] }\end{array}$ & & & & X & & & & & & $x$ \\
\hline $\begin{array}{l}\text { Hinsdale and } \\
\text { colleagues [39] }\end{array}$ & $X$ & $x$ & & & & & & & X & $x$ \\
\hline $\begin{array}{l}\text { Hoyt and } \\
\text { colleagues [26] }\end{array}$ & $x$ & X & $x$ & & & & & & & \\
\hline $\begin{array}{l}\text { Huseynova and } \\
\text { colleagues [40] }\end{array}$ & $x$ & $x$ & $x$ & X & & $x$ & & & $x$ & $x$ \\
\hline $\begin{array}{l}\text { Jacobs and } \\
\text { colleagues [41] }\end{array}$ & & & & X & & & & & $x$ & $x$ \\
\hline $\begin{array}{l}\text { Pierce and } \\
\text { colleagues }[42]^{a}\end{array}$ & $x$ & X & $x$ & X & & $x$ & & & $x$ & $x$ \\
\hline $\begin{array}{l}\text { Piontek and } \\
\text { colleagues [43] }\end{array}$ & $X$ & X & & & & $x$ & & & $x$ & $x$ \\
\hline $\begin{array}{l}\text { Podnos and } \\
\text { colleagues [44] }\end{array}$ & & & $x$ & $x$ & & & & & $x$ & $x$ \\
\hline $\begin{array}{l}\text { Roettger and } \\
\text { colleagues [45] }\end{array}$ & $x$ & X & & X & & & & & & $x$ \\
\hline $\begin{array}{l}\text { Rotondo and } \\
\text { colleagues [46] }\end{array}$ & & & & X & & & & & $x$ & $x$ \\
\hline $\begin{array}{l}\text { Schuerer and } \\
\text { colleagues [47] }\end{array}$ & $x$ & & & X & & & & & & \\
\hline
\end{tabular}


following trauma [1,3-5]. Evaluating complications is therefore essential to improve the quality of care for patients admitted to acute care institutions for traumatic injuries. However, valid and reliable performance indicators are dependent on standardized definitions and rigorous methodological quality $[15,48,49]$.

Standardized definitions should include a consensus on which complications should be used, how they should be defined, and the timing of evaluation $[15,48,49]$. First, the results of this study show that there is currently a lack of consensus on which complications should be used to evaluate trauma care; no single complication was used in more than $70 \%$ of studies, only two out of 22 studies shared the same list of specific complications [26,39], less than one-half gave justifications for the complications they used to evaluate care, and only one study demonstrated the clinical relevance of included complications [36]. This finding is supported by a previous study in a surgical population [14]. Second, less than one-half of studies provided definitions of specific complications, a problem noted by others [14]. Third, outcomes should be evaluated over a fixed period of time and should include early post-discharge data (for example, complications within the first 28 days of injury) [15]. Indeed, research has shown that major complications do occur after discharge from acute care [50]. In our systematic review, all studies evaluated complications from admission to discharge, which may unfairly advantage hospitals discharging patients early. Assessing complications after discharge is challenging but trauma registries may be linked to hospital discharge datasets to obtain information on complications that have led to hospital readmission.

Rigorous methodological quality includes, among other considerations, data quality and appropriate risk adjustment $[15,48]$. In this review, data quality assurance was mentioned in only one study [27] and less than one-third of studies used any type of risk adjustment. The problem of data quality or missing data in retrospective evaluations of hospital complications, underlined in our study, has been raised in previous studies $[12,51,52]$. Authors note that complications which are not recorded either due to differential surveillance or because of poor data quality are assumed to be absent, which leads to an underestimation for the incidence of hospital complications. Robust risk adjustment is essential to valid performance comparisons because of the heterogeneous case mix across trauma centers [15]. Indeed, the risk of complications has been reported to vary according to age, gender, injury severity, and comorbidities [18,53]. Distinguishing preventable from nonpreventable complications is also an important challenge in quality evaluations [48], that was partially addressed by three studies included in this review [26,29,39].

\section{Potential limitations}

The results of this systematic review should be interpreted in light of possible limitations. First, despite the exhaustive nature of our search strategy and very good inter-rater agreement on study eligibility, some studies may have been missed. For feasibility reasons, we restricted our website search to major trauma organizations in North America, Europe, and Australasia. By doing so, we may have missed some local studies based on regional trauma organizations. In the event that studies were missed, we may have overlooked some specific complications. Second, missing information in study reports meant that we did not have information on some study characteristics, definitions, and methodological quality criteria. For the latter, lack of information was assumed to mean that they did not meet the criteria and may have led to an underestimation of methodological quality. However, adequate reporting of important information is a reflection of study quality [19]. Third, complications that have been used for performance evaluation of trauma care may not necessarily represent those that researchers and stakeholders consider to be the most clinically relevant. For example, psychiatric complications were only evaluated in two studies and we did not identify any specific psychiatric complications - such as delirium, which is frequent in trauma populations [54]. In addition, the complications identified in this study are likely to have been heavily influenced by data availability, considering that most were based on retrospectively collected administrative or registry data. This influence highlights the importance of using a consensusbased procedure as well as information from a literature review to identify which complications should be used to evaluate trauma care.

\section{Conclusion}

According to the GRADE criteria, we can make a weak recommendation on three complications that should be used to evaluate the performance of acute care trauma hospitals: pulmonary embolism, deep vein thrombosis, and pneumonia. However, considering the heterogeneity of definitions used, the lack of clinical justification for the choice of specific complications, and the low methodological quality of included studies, further research is needed to develop a valid and reliable performance indicator based on complications that can be used to improve the quality and efficiency of trauma care.

\section{Key messages}

- Evidence in the literature is sufficient to make a weak recommendation on three complications that should be used to evaluate trauma center care; pulmonary embolism, deep vein thrombosis, and pneumonia.

- Definitions of complications are heterogeneous. 
- Studies that use complications to evaluate trauma center care are generally of low methodological quality.

- The choice of complications is rarely justified by clinical criteria.

- Further research is needed to develop a valid and reliable performance indicator based on complications that can be used to improve the quality and efficiency of trauma care.

\section{Additional material}

Additional file 1: Diagram presenting MeSH and keywords used in the Medline search strategy.

\section{Abbreviations}

GRADE: Grading the Quality of Evidence and Strength of Recommendations in ATS Guidelines and Recommendations.

\section{Acknowledgements}

This study was funded by the Canadian Institutes of Health Research: the young investigator award and research grant (to LM), and the Fonds de la Recherche du Québec - Santé: clinician-scientist award (to AFT).

\section{Author details}

'Department of Social and Preventive Medicine, Université Laval, 2325 rue de I'Université, Québec City, Québec, Canada G1V 0A6. ²Department of Critical Care Medicine, Medicine and Community Health Sciences (HTS), Institute for Public Health, University of Calgary, 2500 University Dr. NW, Calgary, Alberta, Canada T2N 1N4. ${ }^{3}$ Axe traumatologie-urgence-soins intensifs, Centre de Recherche FRQ-S du CHA-Hôpital Enfant-Jésus, 1401 18e rue, Quebec City, Québec, Canada G1J 1Z4. ${ }^{4}$ Department of Anesthesiology, Division of Critical Care Medicine, Université Laval, 2325 rue de I'Université, Québec City, Québec, Canada G1V OA6.

\section{Authors' contributions}

LM, HTS, and AFT made substantial contributions to conception and design, acquisition of data, as well as the analysis and interpretation of data. LM, HTS, and AFT were involved in drafting the manuscript or revising it critically for important intellectual content. LM, HTS, and AFT gave final approval of the version to be published. Each author participated sufficiently in the work to take public responsibility for appropriate portions of the content. All authors read and approved the final manuscript.

\section{Competing interests}

The authors declare that they have no competing interests.

Received: 20 June 2012 Revised: 22 August 2012

Accepted: 14 September 2012 Published: 16 October 2012

\section{References}

1. Ingraham AM, Xiong W, Hemmila MR, Shafi S, Goble S, Neal ML, Nathens AB: The attributable mortality and length of stay of traumarelated complications: a matched cohort study. Ann Surg 2010, 252:358-362

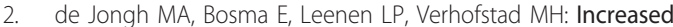
consumption of hospital resources due to complications: an assessment of costs in a level I trauma center. J Trauma 2011, 71:E102-E109.

3. Hemmila MR, Jakubus JL, Maggio PM, Wahl WL, Dimick JB, Campbell DA Jr, Taheri PA: Real money: complications and hospital costs in trauma patients. Surgery 2008, 144:307-316.

4. Shafi $S$, Barnes $S$, Nicewander D, Ballard D, Nathens AB, Ingraham AM, Hemmila M, Goble S, Neal M, Pasquale M, Fildes JJ, Gentilello LM: Health care reform at trauma centers - mortality, complications, and length of stay. J Trauma 2010, 69:1367-1371.

5. O'Keefe GE, Maier RV, Diehr P, Grossman D, Jurkovich GJ, Conrad D: The complications of trauma and their associated costs in a level I trauma center. Arch Surg 1997, 132:920-924, discussion 925.

6. Holbrook TL, Hoyt DB, Anderson JP: The impact of major in-hospital complications on functional outcome and quality of life after trauma. $J$ Trauma 2001, 50:91-95.

7. Reicks $P$, Thorson M, Irwin $E$, Byrnes MC: Reducing complications in trauma patients: use of a standardized quality improvement approach. J Trauma Nurs 2010, 17:185-190.

8. Josten C, Schmidt C: Postoperative complications in trauma surgery Chirurg 2009, 80:790-806.

9. Stelfox H, Straus S, Nathens A, Bobranska-Artiuch B: Evidence for quality indicators to evaluate adult trauma care: a systematic review. Crit Care Med 2011, 39:846-859.

10. Stelfox HT, Bobranska-Artiuch B, Nathens A, Straus SE: Quality indicators for evaluating trauma care: a scoping review. Arch Surg 2010, 145:286-295.

11. Hemmila MR, Nathens AB, Shafi S, Calland JF, Clark DE, Cryer HG, Goble S, Hoeft CJ, Meredith JW, Neal ML, Pasquale MD, Pomphrey MD, Fildes JJ: The Trauma Quality Improvement Program: pilot study and initial demonstration of feasibility. J Trauma 2010, 68:253-262.

12. Hemmila MR, Jakubus JL, Wahl WL, Arbabi S, Henderson WG, Khuri SF, Taheri PA, Campbell DA Jr: Detecting the blind spot: complications in the trauma registry and trauma quality improvement. Surgery 2007, 142:439-448, discussion 448-449.

13. Kamerow D: Quality rankings for US hospitals are released. BMJ 2011, 343:d6539.

14. Martin RC, Brennan MF, Jaques DP: Quality of complication reporting in the surgical literature. Ann Surg 2002, 235:803-813.

15. lezzoni L: Risk Adjustment for Measuring Health Care Outcomes. 3 edition. Chicago: Health Administration Press; 2003.

16. Cochrane Handbook for Systematic Reviews of Interventions. [http:// www.cochrane-handbook.org/]

17. Liberati A, Altman DG, Tetzlaff J, Mulrow C, Gotzsche PC, loannidis JP, Clarke M, Devereaux PJ, Kleijnen J, Moher D: The PRISMA statement for reporting systematic reviews and meta-analyses of studies that evaluate healthcare interventions: explanation and elaboration. BMJ 2009, 339 : b2700.

18. Haider AH, Crompton JG, Oyetunji T, Stevens KA, Efron DT, Kieninger AN, Chang DC, Cornwell EE, Haut ER: Females have fewer complications and lower mortality following trauma than similarly injured males: a risk adjusted analysis of adults in the National Trauma Data Bank. Surgery 2009, 146:308-315

19. von Elm E, Altman DG, Egger M, Pocock SJ, Gotzsche PC, Vandenbroucke JP: The Strengthening the Reporting of Observational Studies in Epidemiology (STROBE) statement: guidelines for reporting observational studies. Ann Intern Med 2007, 147:573-577.

20. Higgins JP, Altman DG, Gotzsche PC, Juni P, Moher D, Oxman AD, Savovic J, Schulz KF, Weeks L, Sterne JA: The Cochrane Collaboration's tool for assessing risk of bias in randomised trials. BMJ 2011, 343:d5928

21. Downs SH, Black N: The feasibility of creating a checklist for the assessment of the methodological quality both of randomised and nonrandomised studies of health care interventions. J Epidemiol Community Health 1998, 52:377-384.

22. Allison PD: Missing Data Thousand Oaks, Sage University, CA; 2001.

23. Hoyt DB, Hollingsworth-Fridlund P, Fortlage D, Davis JW, Mackersie RC: An evaluation of provider-related and disease-related morbidity in a level I university trauma service: directions for quality improvement. J Trauma 1992, 33:586-601.

24. Jaeschke R, Guyatt GH, Dellinger P, Schunemann H, Levy MM, Kunz R, Norris S, Bion J: Use of GRADE grid to reach decisions on clinical practice guidelines when consensus is elusive. BMJ 2008, 337:a744.

25. da Costa BR, Cevallos M, Altman DG, Rutjes AW, Egger M: Uses and misuses of the STROBE statement: bibliographic study. BMJ Open 2011, 1 e000048.

26. Hoyt DB, Coimbra R, Potenza B, Doucet J, Fortlage D, HolingsworthFridlund $\mathrm{P}$, Holbrook T: A twelve-year analysis of disease and provider complications on an organized level I trauma service: as good as it gets? J Trauma 2003, 54:26-36, discussion 36-37. 
27. Ang DN, Rivara FP, Nathens A, Jurkovich GJ, Maier RV, Wang J, MacKenzie EJ: Complication rates among trauma centers. J Am Coll Surg 2009, 209:595-602

28. Calderale SM, Sandru R, Tugnoli G, Di Saverio S, Beuran M, Ribaldi S, Coletti M, Gambale G, Paun S, Russo L, Baldoni F: Comparison of quality control for trauma management between Western and Eastern European trauma centers. World J Emerg Surg 2008, 3:32.

29. Chang DC, Handly N, Abdullah F, Efron DT, Haut ER, Haider AH, Pronovost PJ, Cornwell EE: The occurrence of potential patient safety events among trauma patients: are they random? Ann Surg 2008, 247:327-334.

30. Claridge JA, Schulman AM, Sawyer RG, Ghezel-Ayagh A, Young JS: The 'July phenomenon' and the care of the severely injured patient: fact or fiction? Surgery 2001, 130:346-353.

31. Cohen M, Fath J, Chung R, Ammon A, Matthews J: Impact of a dedicated trauma service on the quality and cost of care provided to injured patients at an urban teaching hospital. J Trauma 1999, 46:1114-1119.

32. Curtis K, Lien D, Chan A, Grove P, Morris R: The impact of trauma case management on patient outcomes. J Trauma 2002, 53:477-482.

33. Davis KA, Cabbad NC, Schuster KM, Kaplan LJ, Carusone C, Leary T, Udelsman R: Trauma team oversight improves efficiency of care and augments clinical and economic outcomes. J Trauma 2008, 65:1236-1242, discussion 1242-1244.

34. DiRusso S, Holly C, Kamath R, Cuff S, Sullivan T, Scharf H, Tully T, Nealon P, Savino JA: Preparation and achievement of American College of Surgeons level I trauma verification raises hospital performance and improves patient outcome. J Trauma 2001, 51:294-299, discussion 299-300.

35. Glance LG, Dick AW, Meredith JW, Mukamel DB: Variation in hospital complication rates and failure-to-rescue for trauma patients. Ann Surg 2011, 253:811-816

36. Haas B, Gomez D, Xiong W, Ahmed N, Nathens AB: External benchmarking of trauma center performance: have we forgotten our elders? Ann Surg 2011, 253:144-150

37. Haut ER, Noll K, Efron DT, Berenholz SM, Haider A, Cornwell EE, Pronovost PJ: Can increased incidence of deep vein thrombosis (DVT) be used as a marker of quality of care in the absence of standardized screening? The potential effect of surveillance bias on reported DVT rates after trauma. J Trauma 2007, 63:1132-1135, discussion 1135-1137.

38. Highstead RG, Johnson LS, Street JH, Trankiem CT, Kennedy SO, Sava JA: July - as good a time as any to be injured. J Trauma 2009, 67:1087-1090

39. Hinsdale JG, Wyatt JO, Stapes L: Change in morbidity patterns after adoption of the American College of Surgeons complication coding system in a regional trauma center: results of a prospective study. $J$ Trauma 1998, 44:821-824, discussion 824-826.

40. Huseynova K Xiong W, Ray JG, Ahmed N, Nathens AB: Venous thromboembolism as a marker of quality of care in trauma. J Am Coll Surg 2009, 208:547-552, 552.e1.

41. Jacobs DG, Sarafin JL, Norton HJ, Christmas AB, Huynh T, Sing RF: Wasted hospital days impair the value of length-of-stay variables in the quality assessment of trauma care. Am Surg 2009, 75:794-802, discussion 802-803.

42. Pierce CA, Haut ER, Kardooni S, Chang DC, Efron DT, Haider A, Pronovost PJ, Cornwell EE: Surveillance bias and deep vein thrombosis in the national trauma data bank: the more we look, the more we find. J Trauma 2008 64:932-936, discussion 936-937.

43. Piontek F, Coscia R, Marselle C, Korn R, Zarling E: Impact of American College of Surgeons verification on trauma outcomes. J Trauma 2003 54:1041-1046, discussion 1046-1047.

44. Podnos YD, Wilson SE, Williams RA: Effect of surgical panel composition on patient outcome at a level I trauma center. Arch Surg 1998, 133:847-854.

45. Roettger RH, Taylor SM, Youkey JR, Blackhurst DW: The general surgery model: a more appealing and sustainable alternative for the care of trauma patients. Am Surg 2005, 71:633-638, discussion 638-639.

46. Rotondo MF, Bard MR, Sagraves SG, Toschlog EA, Schenarts PJ, Goettler CE, Newell MA, Robertson MJ: What price commitment: what benefit? The cost of a saved life in a developing level I trauma center. J Trauma 2009, 67:915-923.

47. Schuerer DJ, Whinney RR, Freeman BD, Nash J, Prasad S, Krem MM, Mazuski JE, Buchman TG: Evaluation of the applicability, efficacy, and safety of a thromboembolic event prophylaxis guideline designed for quality improvement of the traumatically injured patient. J Trauma 2005, 58:731-739.

48. Pronovost PJ, Colantuoni E: Measuring preventable harm: helping science keep pace with policy. JAMA 2009, 301:1273-1275

49. Pronovost PJ, Miller M, Wachter RM: The GAAP in quality measurement and reporting. JAMA 2007, 298:1800-1802.

50. Battistella FD, Torabian SZ, Siadatan KM: Hospital readmission after trauma: an analysis of outpatient complications. J Trauma 1997, 42:1012-1016, discussion 1016-1017.

51. Kardooni S, Haut ER, Chang DC, Pierce CA, Efron DT, Haider AH, Pronovost PJ, Cornwell EE: Hazards of benchmarking complications with the National Trauma Data Bank: numerators in search of denominators. J Trauma 2008, 64:273-277, discussion 277-279.

52. Haut ER, Pronovost PJ: Surveillance bias in outcomes reporting. JAMA 2011, 305:2462-2463

53. de Jongh MA, Bosma $\mathrm{E}$, Verhofstad MH, Leenen LP: Prediction models for complications in trauma patients. Br J Surg 2011, 98:790-796.

54. Pandharipande P, Cotton BA, Shintani A, Thompson J, Pun BT, Morris JA Jr, Dittus R, Ely EW: Prevalence and risk factors for development of delirium in surgical and trauma intensive care unit patients. J Trauma 2008, 65:34-41.

doi:10.1186/cc11680

Cite this article as: Moore et al: Complication rates as a trauma care performance indicator: a systematic review. Critical Care 2012 16:R195.

\section{Submit your next manuscript to BioMed Central and take full advantage of:}

- Convenient online submission

- Thorough peer review

- No space constraints or color figure charges

- Immediate publication on acceptance

- Inclusion in PubMed, CAS, Scopus and Google Scholar

- Research which is freely available for redistribution

Submit your manuscript at www.biomedcentral.com/submit
C) Biomed Central 